\title{
Intra- and interobserver variability of language mapping by navigated transcranial magnetic brain stimulation
}

Nico Sollmann ${ }^{1 \dagger}$, Theresa Hauck ${ }^{1 \dagger}$, Alexander Hapfelmeier ${ }^{2}$, Bernhard Meyer ${ }^{1}$, Florian Ringel ${ }^{1}$ and Sandro M Krieg ${ }^{{ }^{*}}$

\begin{abstract}
Background: Repetitive navigated transcranial magnetic stimulation (rTMS) has been used for studying language organization in healthy volunteers and patients, and to detect cortical areas involved in language processing. However, little is known about the reliability of this method. To determine the reliability of rTMS language mapping, we conducted both an interobserver and an intraobserver investigation.

Methods: Ten right-handed healthy subjects underwent language mapping by rTMS and the same object-naming task three times. Intraobserver and interobserver reliability of seven different error types were tested by two investigators. Analysis was performed blinded to the previous results and stimulated cortical sites.

Results: Overall, the results of both the interobserver and the intraobserver investigations show variable accordance. This is demonstrated by comparing the error rates of all different error types of the three examinations. Considering the most important error type, "no response", there is only small variability in inter- and intraobserver mapping.

Conclusions: With our current protocol, interobserver and intraobserver comparisons only corresponded partially. Thus, although rTMS seems a promising method for preoperative planning as well as neuropsychological research, the current protocol needs further improvement.
\end{abstract}

Keywords: Cortical mapping, Language, Transcranial magnetic stimulation, Navigated brain stimulation, Object naming

\section{Background}

Data on the cortical organization of human language is largely based on functional MRI (fMRI) and magnetoencephalography (MEG) studies [1-6]. Moreover, intraoperative language mapping by bipolar direct cortical stimulation (DCS) during awake surgery for the left [7-9] and right hemispheres [10] also contributed to the current knowledge. Although DCS mapping is highly reliable, it does not allow for analysis of cortical language distribution in the healthy brain. Therefore, as the second lesion-based but non-invasive modality, transcranial magnetic stimulation (TMS) was used since two decades to disrupt language function [11-13]. With

\footnotetext{
* Correspondence: Sandro.Krieg@|rz.tum.de

${ }^{\dagger}$ Equal contributors

'Department of Neurosurgery, Klinikum rechts der Isar, Technische

Universität München, Ismaninger Str. 22, 81675 Munich, Germany

Full list of author information is available at the end of the article
}

the introduction of navigated TMS (nTMS) in neuroscience in the last years repetitive nTMS (rTMS) was also increasingly used for mapping of cortical regions, which are presumably language-eloquent $[11,12,14-16]$.

In combination with an object naming task, rTMS was also compared to intraoperative DCS during awake surgery $[15,16]$. Therefore, this new technique is already seen as a tool for preoperative mapping of cortical language function. However, while mapping of the primary motor cortex by nTMS via provoking motor evoked potentials was already proven to be sufficiently reproducible $[17,18]$, data confirming the reliability of rTMS for language mapping are still lacking.

Given the remaining uncertainty about reproducibility of rTMS language mapping, this study aims to determine the reliability of rTMS language mapping by conducting both an intra- and interobserver investigation. 
To mirror the current practice of rTMS language mapping we strictly adhered to the recently published protocols instead of using a new and more standardized protocol $[15,16,19]$.

\section{Methods}

Study design

The study was designed as prospective, non-randomized.

\section{Study subjects}

Ten purely right-handed (according to the Edinburgh handedness questionnaire), monolingual and healthy volunteers underwent rTMS three times. German was the only primary language for all volunteers. Five subjects were female and five were male. The median age was 24.2 years (range 22.7 to 30.3 years). No one was under any kind of medication.

The inclusion criteria were German as mother tongue, right handedness, written informed consent, and age above18 years. The exclusion criteria were general rTMS exclusion criteria (pacemaker, cochlear implant), previous seizures, second mother tongue, bilateral handedness, and pathological findings on cranial MRI.

\section{Ethics}

The experimental protocol was approved by the ethical committee of the Technical University of Munich (registration number: 2793/10) in accordance with the Declaration of Helsinki. All volunteers provided written informed consent prior to MR imaging.

\section{Navigational MRI}

Prior to the first rTMS mapping session, all volunteers underwent a navigational MRI scan on the same clinical 3 Tesla MR scanner (Achieva 3T, Philips Medical Systems, The Netherlands B.V.) by use of an 8-channel phased array head coil. A 3D gradient echo sequence (TR/TE 9/4 ms, $1 \mathrm{~mm}^{2}$ isovoxel covering the whole head, 6 minutes 58 seconds acquisition time) without intravenous contrast administration was used for anatomical co-registration. Then, by using the DICOM standard, the three-dimensional dataset was transferred to the rTMS system.

\section{rTMS language mapping Experimental setup}

Each subject underwent rTMS language mapping three times by two different investigators. In order to evaluate the intraobserver reliability, the first and the second mapping were conducted by the same investigator. Another investigator performed the third mapping to determine interobserver reliability. The time lag between the first and the second mapping was $191.4 \pm 67.5$ days. Between the first and the third mapping, the time lag was
$211.8 \pm 60.7$ days. Data collection and analysis were performed blinded to previous results, by the same investigator who also performed the mapping.

The experimental setup was strictly the same for all cortical language mappings, which were performed with the Nexstim eXimia NBS system version 4.3 and the NexSpeech ${ }^{\circ}$ module (Nexstim Oy, Helsinki, Finland), as reported earlier $[15,16]$. In short, object presentation was performed by 131 color pictures of common objects, displayed at an inter-stimulus interval (ISI) of $2.5 \mathrm{~s}$ on a video screen in front of the volunteer. The subject was instructed to name the objects in German as quickly and precisely as possible. Magnetic pulses were applied 300 $\mathrm{ms}$ after the picture presentation onset, according to our current knowledge of the timing of naming-related activity reported in MEG and TMS studies $[5,20]$ and according to a protocol established in the first study on navigated TMS for language mapping [14]. First, baseline testing of all objects without stimulation was performed twice prior to each language mapping. All objects, which were misnamed at least once, were discarded from the stimulus sequence and the number of baseline errors was documented. Subsequently, the definite diagnostic naming task was presented timelocked to a train of rTMS pulses. During baseline, as well as during language mapping, minimum electric field strength of $55 \mathrm{~V} / \mathrm{m}$ at the cortical surface was accepted. Overall, field strength ranged between $55-80 \mathrm{~V} / \mathrm{m}$ among all subjects. Frequency and intensity of the rTMS were personalized based on a previously published protocol at $100 \%$ Resting Motor Threshold (RMT) of the left-sided cortical hand area of the right abductor pollicis brevis muscle $[21,22]$. Trains of 5-7 TMS bursts were administered to $\mathrm{vPrG}$ and opIFG with three different setups (a: $5 \mathrm{~Hz}, 5$ pulses; b: $7 \mathrm{~Hz}, 5$ pulses; c: 7 $\mathrm{Hz}, 7$ pulses). Then, the setup $(\mathrm{a}-\mathrm{c})$ that caused the most effective language impairment was used for further mapping. If there was no clear difference in the effect on language the most comfortable frequency was chosen. If no evident responses were obtained, the intensity was increased to $110-120 \%$ RMT and we started again with setup a-c. For objective and detailed analysis, the object naming baseline performance, as well as the mapping session, were digitally video-recorded $[14,15]$.

\section{Language mapping procedure}

The stimulation coil was randomly moved during the ISI in $10 \mathrm{~mm}$ steps over the left hemisphere. In order to achieve maximum field induction, the coil was placed tangential to the skull and with the field in an anteriorposterior orientation, as reported earlier [12,14,23]. All sites were stimulated three times each, and the same sites were not targeted consecutively. Language mapping of the left hemisphere required up to 60 minutes per 
subject. Directly after each mapping, each volunteer was asked to determine the rate of discomfort or pain according to the visual analogue scale (VAS) from 0 (no pain) to 10 (maximum pain) both for temporal regions and for the convexity (defined as the lateral, dorsal, rostral, and cranial surface of the brain without the region covered by the temporal muscle).

\section{Data analysis}

The mapping data of all subjects were examined as previously described $[14,15]$. The first two mappings of each volunteer were analyzed by the same examiner who had also performed the corresponding stimulation sessions. The third mapping was evaluated by the second examiner who conducted the third mapping sessions in an analogue way. The comparison between the first and second mapping sessions was intended to gauge intraobserver variability, while the comparison between the second and third mapping sessions measures interobserver variability. Analysis was performed strictly blinded to the previous results and stimulated cortical sites.

Prior to video analysis, the individual RMT and rTMS frequencies were documented. Then, the video was analyzed for any disturbance of language processing compared to the baseline response. During video analysis, the cortical stimulation sites were hidden. Errors related to direct stimulation of muscles or associated with pain were discarded from the analysis. All other observed errors were categorized following a rule presented in Corina et al. [24]:

No-response errors: stimulation leads to a complete lack of naming response.

Performance errors: form-based distortions that are slurred, stuttered, or imprecisely articulated. This category contains both dysarthric and apraxic language production errors.

Hesitation: delayed onset of naming compared to baseline.

Neologisms: form-based errors, which are possible but nonexistent words. For example, the target word "horse" is replaced with the word "herp".

Semantic paraphasias: errors in which the volunteer substitutes a semantically related or associated word for the target word. For example, the target word "cow" is replaced by the word "horse".

Phonologic paraphasias: characterized by unintended phonemic modification of the target word. The spoken word resembles the target word, but is phonetically different. For example, the target word "pants" is replaced with "plants".

Circumlocution errors: errors in which the subject talks about or "around" the target, instead of naming it.
For example, the target word "chair" is replaced with "sit down", explaining the use of the target word.

\section{Frequency map of evoked errors Anatomical localization}

The location of stimulation in the rTMS system was determined with a real time physics-modeling system (eXimia 4.3, Nexstim Oy, Helsinki, Finland). This system calculates the intracranial location of stimulation induced by the coil and displays this information on a high-resolution 3D MRI. As a result, the effects of the stimulation train can be pinpointed to an anatomical location when the coil position is tracked. According to an approach for evaluating effects of intraoperative language mapping on an anatomical level, we used the cortical parcellation system (CPS) for further anatomyrelated data analysis and visualization $[24,25]$. The CPS divides the cortex into 37 anatomical regions for evaluation of the anatomical site of the stimulation (Table 1). The cortical gyri belonging to these anatomical CPS subregions were identified from 3D MRIs and the regions were drawn on a $3 \mathrm{D}$ image [25]. Then, the rTMS data were projected on the parcellated cortex. This approach allows a statistical analysis of error frequency and comparison of the data, both between individual volunteers and over the entire studied population. The locations of these regions were displayed on an anatomical brain template (Table 1, Figure 1).

Additionally, for combined statistical and anatomical analysis, two parcellation groups were formed:

(1) Anterior group: including CPS regions opIFG, trIFG, and $v \operatorname{PrG}$

(2) Posterior group: including CPS regions AnG, aSMG, pSMG, mSTG, and pSTG

\section{Stimulation assessment}

As aforementioned, each stimulation site was stimulated three times during rTMS language mapping. To determine whether an individual brain region gave rise to language deficits during rTMS or not, the following definitions for region positivity and negativity were used:

(1) Positive brain region: a region was considered to give rise to language deficits if any of the trains delivered to the region elicited naming errors, regardless of the error type; and,

(2) Negative brain region: a brain region was considered not to give rise to language deficits if the region had been stimulated with at least one stimulation train and no language deficits of any error type were generated. 
Table 1 Cortical parcellation system

\begin{tabular}{|c|c|}
\hline Abbreviation & Anatomy \\
\hline alTG & Anterior inferior temporal gyrus \\
\hline aMFG & Anterior middle frontal gyrus \\
\hline aMTG & Anterior middle temporal gyrus \\
\hline anG & Angular gyrus \\
\hline aSFG & Anterior superior frontal gyrus \\
\hline aSMG & Anterior supramarginal gyrus \\
\hline aSTG & Anterior superior temporal gyrus \\
\hline dLOG & Dorsal lateral occipital gyrus \\
\hline dPoG & Dorsal post-central gyrus \\
\hline dPrG & Dorsal pre-central gyrus \\
\hline mITG & Middle inferior temporal gyrus \\
\hline mMFG & Middle middle frontal gyrus \\
\hline mMTG & Middle middle temporal gyrus \\
\hline $\mathrm{mPoG}$ & Middle post-central gyrus \\
\hline $\mathrm{mPrG}$ & Middle pre-central gyrus \\
\hline mSFG & Middle superior frontal gyrus \\
\hline mSTG & Middle superior temporal gyrus \\
\hline oplFG & Opercular inferior frontal gyrus \\
\hline orlFG & Orbital part of the inferior frontal gyrus \\
\hline plTG & Posterior inferior temporal gyrus \\
\hline pMFG & Posterior middle frontal gyrus \\
\hline pMTG & Posterior middle temporal gyrus \\
\hline pollFG & Polar inferior frontal gyrus \\
\hline pollTG & Polar inferior temporal gyrus \\
\hline polLOG & Polar lateral occipital gyrus \\
\hline polMFG & Polar middle frontal gyrus \\
\hline polMTG & Polar middle temporal gyrus \\
\hline polSFG & Polar superior frontal gyrus \\
\hline polsTG & Polar superior temporal gyrus \\
\hline pSFG & Posterior superior frontal gyrus \\
\hline pSMG & Posterior supramarginal gyrus \\
\hline pSTG & Posterior superior temporal gyrus \\
\hline SPL & Superior parietal lobe \\
\hline $\operatorname{trlFG}$ & Triangular inferior frontal gyrus \\
\hline vLOG & Ventral lateral occipital gyrus \\
\hline vPoG & Ventral post-central gyrus \\
\hline vPrG & Ventral pre-central gyrus \\
\hline
\end{tabular}

Anatomical names and abbreviations according to Corina et al. [25].

\section{Statistics}

Descriptive and explorative statistics were used for data analysis. The distribution of quantitative data is presented by the mean \pm standard deviation. Differences concerning the determined RMT, the mapping intensity, and the indicated pain during the three mappings were tested via analysis of variance (ANOVA) for repeated measures (SigmaStat 3.5, Jandel Scientific, Erkrath, Germany). Additionally, a permutation test of symmetry was performed to evaluate differences in the utilized frequencies and pulses (The R Foundation for Statistical Computing, Vienna, Austria). A $P$ value of $P<0.05$ was considered significant.

The concordance correlation coefficient (CCC) was used to compute intra- and inter-observer reliability (The R Foundation for Statistical Computing, Vienna, Austria) [26]. For the former, two assessments of one observer were compared. For the latter, the first measurements of observer one were compared against the ones of observer two.

The CCC measures the agreement between two variables. Perfect agreement means a CCC of 1.

\section{Results}

Healthy subjects

Altogether, 10 volunteers were enrolled (Table 2). No volunteer had to be excluded due to intracranial pathologies.

\section{Stimulation-related discomfort}

Each stimulation session was generally well tolerated by the volunteers and no volunteers requested reduction of the stimulation intensity due to pain. The mean VAS score for maximum painful stimuli was comparable in both groups (Table 2). However, given experiences in former studies, the spatial extent of stimulation had to be restricted because of unacceptable pain in orIFG, polSTG, polMTG, and aMTG. In addition, the ITG was not mapped due to the distance between skin and brain, and the consequent too-low stimulation intensity.

\section{Distribution of the different error types First mapping}

Sum of all errors Overall, 3606 stimulations were performed. Of these, 961 stimulations elicited a language error. This equals an error rate of $26.7 \%$ (Table 3). The errors were mainly located within the classical Broca's area of the opIFG and its surrounding structures. However, when also considering dysarthria, vPrG and $\mathrm{mPrG}$ showed relatively high error rates (Figure 2).

No response errors Of 3606 left hemispheric stimulations, 179 no response errors occurred. This is equal to an error rate of $5.0 \%$ (Table 3). No response errors were observed in all subjects during the first mapping session.

Performance errors Of the 3606 stimulations, 410 performance errors were observed, which is equal to an error rate of $11.4 \%$ (Table 3). Each volunteer showed performance errors. 


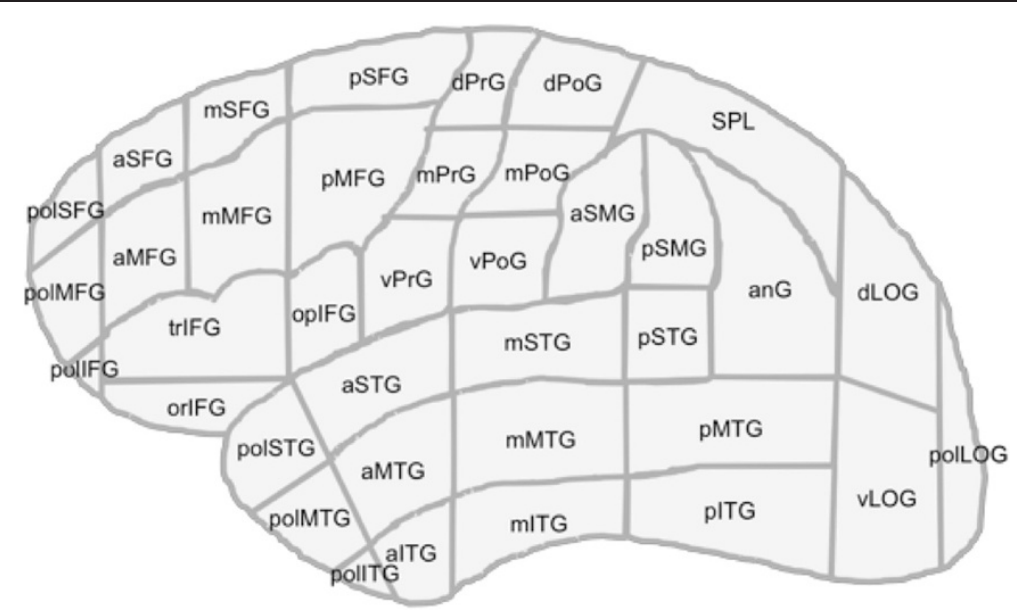

Figure 1 Cortical parcellation system. Anatomical areas, as described in Corina et al. [25].

Hesitation errors Hesitation errors as an isolated error type were very widely distributed and were observed in all volunteers; 294 hesitation errors were found out of 3606 stimulations, which is equal to an error rate of $8.2 \%$ (Table 3).

Other errors Out of all 3606 stimulations, 62 neologisms (error rate 1.7\%), and 4 phonological errors (error rate $0.1 \%$ ) occurred (Table 3 ). There were also 12 semantic errors (error rate $0.3 \%$ ). No circumlocutions were observed.

\section{Second mapping}

Sum of all errors During the second mapping sessions, the left hemisphere was stimulated between 279 and 348 sites (median: 297.0 sites). Across all subjects, 3033 total stimulations were performed in the left hemisphere. Of these, 705 stimulations elicited a language error, for an error rate of $23.2 \%$ (Table 4). These errors were again mainly located within the classical Broca's area of the opIFG, as well its surrounding structures. Again, with regard to elucidating dysarthria, $\mathrm{vPrG}$ and $\mathrm{MPrG}$ showed relatively high error rates (Figure 2).

No response errors Altogether, 116 no response errors occurred. For the number of given stimulations, we obtained an error rate of $3.8 \%$ (Table 4). In the second mapping, no response errors were observed in all subjects as well.

Performance errors Within all volunteers, 241 performance errors were elicited during 3033 stimulations, resulting in an error rate of $7.9 \%$ (Table 4). Again, each volunteer presented performance errors.

Hesitation errors 320 hesitation errors were elicited, for an error rate of $10.6 \%$ (Table 4). Hesitation errors were observed in all subjects.

Other errors With regard to all volunteers, 3033 left hemispheric stimulations elicited 15 neologisms (error rate $0.5 \%$ ) and 13 semantic errors (error rate $0.4 \%$ ). Neither phonological errors nor circumlocutions were observed (Table 4).

Table 2 Stimulation parameters

\begin{tabular}{|c|c|c|c|c|c|}
\hline & & 1st mapping & 2nd mapping & 3rd mapping & $\mathbf{p}$ \\
\hline Pain (VAS) & convexity & $1.7 \pm 1.2$ & $2.1 \pm 1.2$ & $2.0 \pm 1.2$ & 0.662 \\
\hline$($ Mean $\pm S D)$ & temporal & $5.5 \pm 1.6$ & $5.7 \pm 1.8$ & $4.8 \pm 1.5$ & 0.318 \\
\hline \multicolumn{2}{|c|}{ motor threshold (\% Output) (mean \pm SD) } & $37.8 \pm 7.0$ & $36.7 \pm 5.4$ & $35.9 \pm 7.1$ & 0.439 \\
\hline \multicolumn{2}{|c|}{ mapping intensity $(\% \mathrm{MT})($ mean \pm SD) } & $102 \pm 6$ & $104 \pm 8$ & $102 \pm 6$ & 0.685 \\
\hline \multirow[t]{3}{*}{ most comfortable } & $5 \mathrm{~Hz}, 5$ Pulses & 5 & 3 & 2 & 0.233 \\
\hline & $7 \mathrm{~Hz}, 5$ Pulses & 2 & 6 & 6 & \\
\hline & $7 \mathrm{~Hz}, 7$ Pulses & 3 & 1 & 2 & \\
\hline
\end{tabular}


Table 3 First mapping

\begin{tabular}{|c|c|c|c|c|c|c|c|c|c|c|c|c|c|c|c|c|c|}
\hline \multirow{2}{*}{ Subject } & \multicolumn{2}{|c|}{ No response } & \multicolumn{2}{|c|}{ Performance } & \multicolumn{2}{|c|}{ Hesitation } & \multicolumn{2}{|c|}{ Neologism } & \multicolumn{2}{|c|}{ Phonological } & \multicolumn{2}{|c|}{ Circumlocution } & \multicolumn{2}{|c|}{ Semantic } & \multicolumn{3}{|c|}{ Totals } \\
\hline & Errors & Rate & Errors & Rate & Errors & Rate & Errors & Rate & Errors & Rate & Errors & Rate & Errors & Rate & Errors & Trials & Error rate \\
\hline M1 & 28 & 0.05 & 124 & 0.21 & 40 & 0.07 & 48 & 0.08 & 0 & 0.00 & 0 & 0.00 & 1 & 0.00 & 241 & 591 & 0.41 \\
\hline M2 & 17 & 0.06 & 28 & 0.10 & 32 & 0.11 & 0 & 0.00 & 0 & 0.00 & 0 & 0.00 & 0 & 0.00 & 77 & 294 & 0.26 \\
\hline M3 & 22 & 0.06 & 42 & 0.12 & 32 & 0.09 & 5 & 0.01 & 0 & 0.00 & 0 & 0.00 & 2 & 0.01 & 103 & 363 & 0.28 \\
\hline M4 & 5 & 0.02 & 32 & 0.11 & 20 & 0.07 & 3 & 0.01 & 1 & 0.00 & 0 & 0.00 & 2 & 0.01 & 63 & 300 & 0.21 \\
\hline M5 & 2 & 0.01 & 51 & 0.17 & 14 & 0.05 & 3 & 0.01 & 2 & 0.01 & 0 & 0.00 & 0 & 0.00 & 72 & 300 & 0.24 \\
\hline $\mathrm{F} 1$ & 17 & 0.06 & 37 & 0.13 & 22 & 0.08 & 2 & 0.01 & 0 & 0.00 & 0 & 0.00 & 2 & 0.01 & 80 & 291 & 0.27 \\
\hline F2 & 20 & 0.05 & 31 & 0.08 & 34 & 0.09 & 0 & 0.00 & 0 & 0.00 & 0 & 0.00 & 0 & 0.00 & 85 & 366 & 0.23 \\
\hline F3 & 10 & 0.03 & 19 & 0.05 & 32 & 0.09 & 1 & 0.00 & 0 & 0.00 & 0 & 0.00 & 1 & 0.00 & 63 & 354 & 0.18 \\
\hline F4 & 16 & 0.04 & 35 & 0.08 & 21 & 0.05 & 0 & 0.00 & 1 & 0.00 & 0 & 0.00 & 2 & 0.00 & 75 & 450 & 0.17 \\
\hline F5 & 42 & 0.14 & 11 & 0.04 & 47 & 0.16 & 0 & 0.00 & 0 & 0.00 & 0 & 0.00 & 2 & 0.01 & 102 & 297 & 0.34 \\
\hline MIN & 2 & 0.01 & 11 & 0.04 & 14 & 0.05 & 0 & 0.00 & 0 & 0.00 & 0 & 0.00 & 0 & 0.00 & 63 & 291 & 0.17 \\
\hline MAX & 42 & 0.14 & 124 & 0.21 & 47 & 0.16 & 48 & 0.08 & 2 & 0.01 & 0 & 0.00 & 2 & 0.01 & 241 & 591 & 0.41 \\
\hline MEDIAN & 17 & 0.05 & 33.5 & 0.11 & 32 & 0.09 & 1.5 & 0.01 & 0 & 0.00 & 0 & 0.00 & 1.5 & 0.00 & 78.5 & 327 & 0.25 \\
\hline
\end{tabular}

Distribution of naming errors in the first mapping: Summary of naming errors induced by rTMS per subject (M1-M5: male volunteers; F1-F5: female volunteers). Below is reported number of trials (rTMS trains), total number errors, and error type and rate of each given error type of all induced errors.

\section{Third mapping}

Sum of all errors The left hemisphere was stimulated between 384 and 477 sites (median: 436.5 sites) during the third mapping sessions. With 4344 total stimulations, the highest number of stimulations was set during these third mapping sessions. Thereby, 745 language errors were elicited (error rate 17.2\%; Table 5). Once again, these errors of any kind were primarily located within the classical Broca's area of the opIFG, as well its surrounding structures (Figure 2).

No response errors 148 no response errors were elicited by 4344 stimulations in the left hemisphere. This equals an error rate of $3.4 \%$ (Table 5). Again, no response errors were observed in each subject.

Performance errors In contrast to the previous mappings, only 8 out of 10 subjects showed performance errors. Altogether, 61 performance errors were elicited, resulting in an error rate of $1.4 \%$ (Table 5).

Hesitation errors Hesitation errors came up to a number of 492. Consequentially, this represents an error rate of $11.3 \%$ (Table 5). Hesitations were again observed in all subjects.

Other errors 4344 left hemispheric stimulations elicited 24 neologisms (error rate $0.6 \%$ ) and 13 phonological errors (error rate 0.3\%). Furthermore, we obtained 7 semantic errors (error rate $0.2 \%$; Table 5). Again, no circumlocutions were observed.
Intra- and interobserver reliability

The properties of all three mappings are demonstrated by Table 2. Differences among the mapping parameters did not reach statistical significance.

\section{Intraobserver reliability}

To determine the intraobserver reliability, the first two mappings, which were conducted by the same examiner, were compared. The reproducibility varied among different error categories. Overall, among intraobserver investigations, no response errors, hesitations, and semantic errors were better reproducible than other error categories. Posterior regions showed lower reproducibility than the anterior regions. Table 6 demonstrates the results expressed by the CCC.

\section{Interobserver reliability}

To determine the interobserver reliability, the first and third mapping, which were conducted by two different examiners, were compared. Again, we obtained higher reproducibility in anterior regions and in the error categories of "no response", "hesitations", and "semantic errors". Altogether, performance errors and phonological errors showed-as in intraobserver investigations-low reproducibility (Table 6).

\section{Discussion}

During all mapping sessions, and given our previously defined spatial limitations, rTMS was well tolerated by all volunteers. There were neither adverse events nor statistically significant differences concerning the experienced pain. Within our small study group, we conclude 


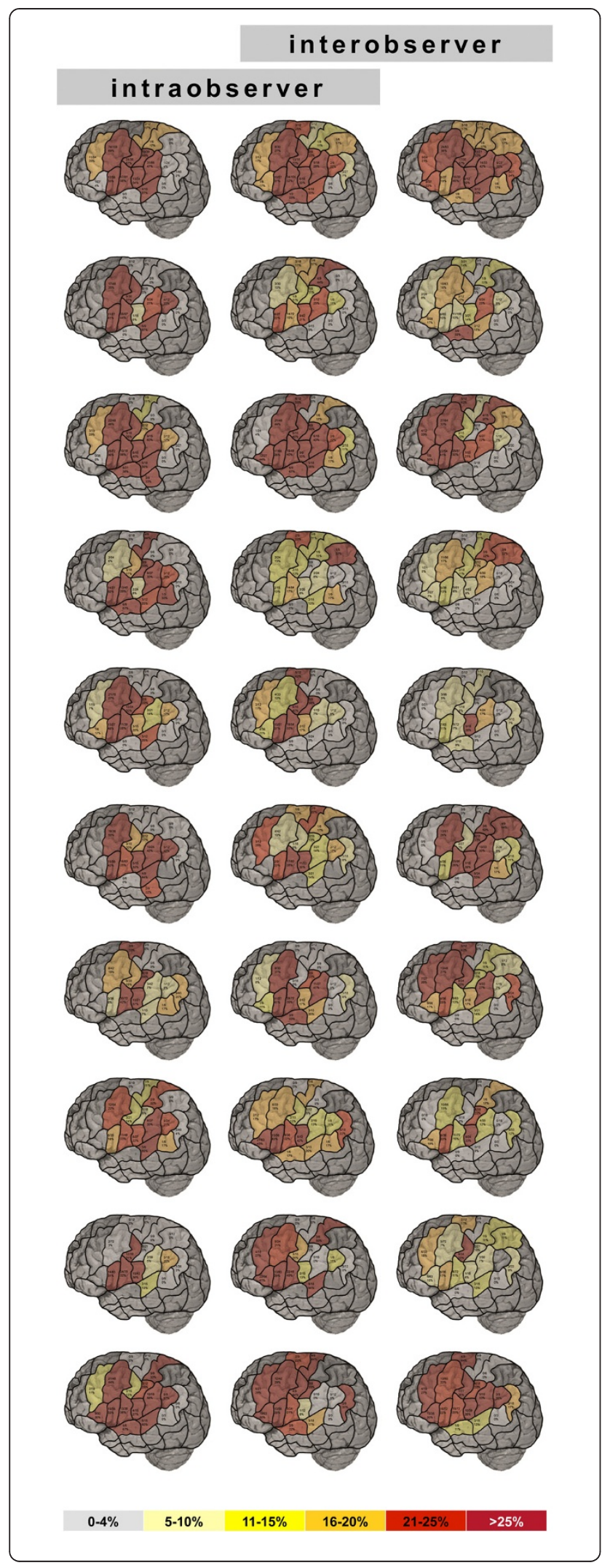

Figure 2 All errors. Language mapping by rTMS presented with the cortical parcellation system. Each line shows the results for all errors of one subject. The left and the intermediate column illustrate intraoberserver, the intermediate and the right column illustrate interobserver variability.

that intraprocedural discomfort is not related to the investigator.

The technique of rTMS is a promising new method for preoperative planning of surgery in eloquent cortical areas. In clinical daily routines, it is common practice that a certain examination method is conducted by different investigators. Therefore, high reliability of the respective method is necessary. The authors decided to conduct the remappings blinded to previous mapping setups (frequency, number of pulses, RMT) and results, to both mirror clinical reality and to include the entire rTMS procedure. Our data showed that the rTMS language mapping has limited reliability not only in intra-observer comparisons, but also in interobserver comparisons; although there is not a perfect match, there is a reasonably small range of differences.

The observed variability is ascribable to at least three different causes, though the abovementioned design of the present study does not allow us to determine which of the following three variables has the most important influence on the observed intra- and interobserver variability. First, differences in the performance of the mappings, such as applying distinct mapping parameters, probably lead to different language positive points. Furthermore, reproducibility can also be impaired by the language analysis itself because analyzing the recorded videos is not completely objective. Although our setup with baseline measurements, standardized protocols, and video recording for detailed post-hoc analysis of the language responses reduces a lot of bias, the evaluation of language errors still harbors some subjective issues. Additionally, we have to proceed on the assumption that there exists no absolute stability in the organization of human language function in the brain, which also has a conceivably significant influence on variability in language mapping $[27,28]$.

With regard to the different mapping parameters as a source of mapping differences, the investigators strictly followed the mapping protocol mentioned above during all examinations. According to our mapping protocol, it remains to the investigator to choose between three different setups $(5 \mathrm{~Hz} / 5$ pulses, $5 \mathrm{~Hz} / 7$ pulses, or $7 \mathrm{~Hz} / 7$ pulses). Furthermore, the hand knob and the RMT were determined again for each remapping. However, the chosen mapping parameters and the detected RMT show no significant difference concerning both intra- and interobserver investigations (Table 2). Hence, we assume that the choice of parameters is distributed randomly. In 
Table 4 Second mapping

\begin{tabular}{|c|c|c|c|c|c|c|c|c|c|c|c|c|c|c|c|c|c|}
\hline \multirow{2}{*}{ Subject } & \multicolumn{2}{|c|}{ No response } & \multicolumn{2}{|c|}{ Performance } & \multicolumn{2}{|c|}{ Hesitation } & \multicolumn{2}{|c|}{ Neologism } & \multicolumn{2}{|c|}{ Phonological } & \multicolumn{2}{|c|}{ Circumlocution } & \multicolumn{2}{|c|}{ Semantic } & \multicolumn{3}{|c|}{ Totals } \\
\hline & Errors & Rate & Errors & Rate & Errors & Rate & Errors & Rate & Errors & Rate & Errors & Rate & Errors & Rate & Errors & Trials & Error rate \\
\hline M1 & 17 & 0.05 & 34 & 0.10 & 41 & 0.12 & 4 & 0.01 & 0 & 0.00 & 0 & 0.00 & 0 & 0.00 & 96 & 345 & 0.28 \\
\hline M2 & 11 & 0.04 & 7 & 0.03 & 28 & 0.10 & 0 & 0.00 & 0 & 0.00 & 0 & 0.00 & 0 & 0.00 & 46 & 279 & 0.16 \\
\hline M3 & 25 & 0.07 & 29 & 0.08 & 50 & 0.14 & 4 & 0.01 & 0 & 0.00 & 0 & 0.00 & 4 & 0.01 & 112 & 348 & 0.32 \\
\hline M4 & 2 & 0.01 & 18 & 0.06 & 12 & 0.03 & 1 & 0.00 & 0 & 0.00 & 0 & 0.00 & 1 & 0.00 & 34 & 288 & 0.12 \\
\hline M5 & 7 & 0.02 & 32 & 0.11 & 15 & 0.05 & 3 & 0.01 & 0 & 0.00 & 0 & 0.00 & 1 & 0.00 & 58 & 288 & 0.20 \\
\hline F1 & 4 & 0.01 & 26 & 0.09 & 22 & 0.08 & 0 & 0.00 & 0 & 0.00 & 0 & 0.00 & 1 & 0.00 & 53 & 282 & 0.19 \\
\hline F2 & 10 & 0.03 & 18 & 0.06 & 43 & 0.14 & 0 & 0.00 & 0 & 0.00 & 0 & 0.00 & 0 & 0.00 & 71 & 303 & 0.23 \\
\hline F3 & 11 & 0.04 & 21 & 0.07 & 40 & 0.13 & 3 & 0.01 & 0 & 0.00 & 0 & 0.00 & 4 & 0.01 & 79 & 306 & 0.26 \\
\hline F4 & 13 & 0.04 & 42 & 0.14 & 27 & 0.09 & 0 & 0.00 & 0 & 0.00 & 0 & 0.00 & 1 & 0.00 & 83 & 297 & 0.28 \\
\hline F5 & 16 & 0.05 & 14 & 0.05 & 42 & 0.14 & 0 & 0.00 & 0 & 0.00 & 0 & 0.00 & 1 & 0.00 & 73 & 297 & 0.25 \\
\hline MIN & 2 & 0.01 & 7 & 0.03 & 12 & 0.03 & 0 & 0.00 & 0 & 0.00 & 0 & 0.00 & 0 & 0.00 & 34 & 279 & 0.12 \\
\hline MAX & 25 & 0.07 & 42 & 0.14 & 50 & 0.14 & 4 & 0.01 & 0 & 0.00 & 0 & 0.00 & 4 & 0.01 & 112 & 348 & 0.32 \\
\hline MEDIAN & 11 & 0.04 & 23.5 & 0.08 & 34 & 0.11 & 0.5 & 0.00 & 0 & 0.00 & 0 & 0.00 & 1 & 0.00 & 72 & 297 & 0.24 \\
\hline
\end{tabular}

Distribution of naming errors in the second mapping (first remapping): Summary of naming errors induced by rTMS per subject (M1-M5: male volunteers; F1-F5: female volunteers). Below is reported number of trials (rTMS trains), total number errors, and error type and rate of each given error type of all induced errors.

other words, none of the examiners seems to prefer a certain mapping setup. Thus, these data suggest that the optimal mapping setup varies considerably even for one subject across the three sessions, yielding a small variability in mapping outcomes.

Concerning the naming error analysis as a source of mapping difference, on one hand, by categorizing the produced errors into pre-defined different error types, the language analysis reaches a certain degree of objectivity. As a result, both the intra- and interobserver variability that may arise at the stage of error analysis could be considered reasonably small. On the other hand, there are still cases in which the investigators are not in accordance. Consequently, it is reasonable that our results demonstrate higher intraobserver compared to interobserver reliability (Figure 2).

When we take a closer look at the intra- and interobserver variability by error types, they were not uniform. Hesitation errors show-besides no response and semantic errors-a high reproducibility. In the existing literature, hesitation errors are suspected to represent a rather untrustworthy error category, which some authors do not even implicate in their analysis [14]. However, our data support the argument that hesitation errors

Table 5 Third mapping

\begin{tabular}{|c|c|c|c|c|c|c|c|c|c|c|c|c|c|c|c|c|c|}
\hline \multirow{2}{*}{ Subject } & \multicolumn{2}{|c|}{ No response } & \multicolumn{2}{|c|}{ Performance } & \multicolumn{2}{|c|}{ Hesitation } & \multicolumn{2}{|c|}{ Neologism } & \multicolumn{2}{|c|}{ Phonological } & \multicolumn{2}{|c|}{ Circumlocution } & \multicolumn{2}{|c|}{ Semantic } & \multicolumn{3}{|c|}{ Totals } \\
\hline & Errors & Rate & Errors & Rate & Errors & Rate & Errors & Rate & Errors & Rate & Errors & Rate & Errors & Rate & Errors & Trials & Error rate \\
\hline M1 & 31 & 0.08 & 40 & 0.10 & 46 & 0.12 & 11 & 0.03 & 2 & 0.01 & 0 & 0.00 & 1 & 0.00 & 131 & 384 & 0.34 \\
\hline M2 & 9 & 0.02 & 2 & 0.00 & 44 & 0.09 & 0 & 0.00 & 0 & 0.00 & 0 & 0.00 & 1 & 0.00 & 56 & 468 & 0.12 \\
\hline M3 & 30 & 0.07 & 6 & 0.01 & 66 & 0.15 & 3 & 0.01 & 1 & 0.00 & 0 & 0.00 & 1 & 0.00 & 107 & 441 & 0.24 \\
\hline M4 & 3 & 0.01 & 2 & 0.00 & 35 & 0.07 & 1 & 0.00 & 0 & 0.00 & 0 & 0.00 & 2 & 0.00 & 43 & 468 & 0.09 \\
\hline M5 & 2 & 0.00 & 2 & 0.00 & 21 & 0.05 & 5 & 0.01 & 0 & 0.00 & 0 & 0.00 & 0 & 0.00 & 30 & 450 & 0.07 \\
\hline $\mathrm{F} 1$ & 22 & 0.06 & 0 & 0.00 & 62 & 0.16 & 1 & 0.00 & 0 & 0.00 & 0 & 0.00 & 0 & 0.00 & 85 & 393 & 0.22 \\
\hline F2 & 19 & 0.05 & 0 & 0.00 & 62 & 0.15 & 0 & 0.00 & 0 & 0.00 & 0 & 0.00 & 0 & 0.00 & 81 & 420 & 0.19 \\
\hline F3 & 11 & 0.03 & 1 & 0.00 & 44 & 0.11 & 1 & 0.00 & 0 & 0.00 & 0 & 0.00 & 0 & 0.00 & 57 & 411 & 0.14 \\
\hline F4 & 9 & 0.02 & 2 & 0.00 & 42 & 0.09 & 0 & 0.00 & 0 & 0.00 & 0 & 0.00 & 0 & 0.00 & 53 & 477 & 0.11 \\
\hline $\mathrm{F} 5$ & 12 & 0.03 & 6 & 0.01 & 70 & 0.16 & 2 & 0.00 & 10 & 0.02 & 0 & 0.00 & 2 & 0.00 & 102 & 432 & 0.24 \\
\hline MIN & 2 & 0.00 & 0 & 0.00 & 21 & 0.05 & 0 & 0.00 & 0 & 0.00 & 0 & 0.00 & 0 & 0.00 & 30 & 384 & 0.07 \\
\hline MAX & 31 & 0.08 & 40 & 0.10 & 70 & 0.16 & 11 & 0.03 & 10 & 0.02 & 0 & 0.00 & 2 & 0.00 & 131 & 477 & 0.34 \\
\hline MEDIAN & 11.5 & 0.03 & 2 & 0.00 & 45 & 0.12 & 1 & 0.00 & 0 & 0.00 & 0 & 0.00 & 0.5 & 0.00 & 69 & 436.5 & 0.17 \\
\hline
\end{tabular}

Distribution of naming errors in the third mapping (second remapping): Summary of naming errors induced by rTMS per subject (M1-M5: male volunteers; F1-F5: female volunteers). Below is reported number of trials (rTMS trains), total number errors, and error type and rate of each given error type of all induced errors. 
Table 6 Concordance correlation coefficient

\begin{tabular}{|c|c|c|c|c|c|c|}
\hline \multirow[t]{2}{*}{ Error category } & \multicolumn{2}{|c|}{ All regions } & \multicolumn{2}{|c|}{ Anterior regions } & \multicolumn{2}{|c|}{ Posterior regions } \\
\hline & Intra-observer & Inter-observer & Intra-observer & Inter-observer & Intra-observer & Inter-observer \\
\hline no response & 0.356 & 0.227 & 0.505 & 0.147 & -0.008 & 0.038 \\
\hline performance error & -0.047 & 0.163 & 0.251 & 0.049 & 0.370 & -0.008 \\
\hline hesitation error & 0.383 & 0.312 & 0.288 & 0.162 & 0.173 & 0.161 \\
\hline neologism & -0.035 & 0.559 & 0.189 & 0.238 & 0.139 & 0.588 \\
\hline phonological error & - & -0.135 & - & -0.292 & - & - \\
\hline semantic error & 0.218 & 0.296 & -0.222 & 0.117 & 0.038 & - \\
\hline
\end{tabular}

Intra- and interobserver reliability in all areas, in anterior and in posterior regions, expressed by the CCC.

evoked by rTMS should not be ignored; instead, they should be regarded as evidence for disrupted language processing in the brain [1], as is the case with nonnavigated rTMS studies that standardly measure naming latency difference to localize specific language functions in healthy adult brains [6].

Another important aspect is the fact that little is known about the stability of language eloquent cortical regions per se. Direct cortical stimulation-the current gold standard for language mapping-assumes that the same stimulated areas do not evoke errors in that region consistently [7]. In the standard DCS language mapping procedure, a cortical site is judged language positive when 2 out of 3 stimulations elicit language errors $[9,15]$. This $66 \%$ criterion means that, even within a very short time lag, absolute reproducibility is impossible even for the gold standard, due to the complex connectivity and therefore plasticity of language function. Furthermore, we have to keep in mind previous studies, which have shown that reorganization of the brain exists not only after strokes or in the course of tumor disease, but also in healthy subjects [29-31].

This natural plasticity may not only be demonstrated for rolandic regions in short-term motor-learning experiments [30,32], cognition, and memory structures in longitudinal real-life extensive subjects learning situation [33], but it also appears in perisylvian eloquent areas and inferior parietal cortex for language perception and memory in a longitudinal code-deciphering learning study. According to the long time lag between first and second or third mapping, plasticity might indeed be a reason for the varying results in the present study. Thus, both intra- and interobserver variability are inevitable.

Additionally, when we take a closer look at the variability by error types, we noticed that, given our protocol, certain error types are better reproducible than others are Table 6). Altogether, in comparison to performance errors, neologisms, and phonological errors, the error categories "no response", "hesitations", and "semantic" tend to show higher reproducibility (Table 6). It could be possible that errors that are associated with pronunciation and language production itself exhibit greater fluctuation.
Another explanation could be that, as reported earlier [15], rTMS following the abovementioned mapping protocol seems to be especially useful in anterior sites (Table 6). Compared with posterior sites, anterior language areas tend to demonstrate higher reproducibility and correlation with intraoperative DCS. When reviewing the literature on human language processing, activation of posterior language sites seems to be earlier than $300 \mathrm{~ms}[1,6]$. Therefore, rTMS pulses $300 \mathrm{~ms}$ after picture presentation might be too late to disrupt posterior language processing. Thus, when improving the protocol in future investigations, one of the questions we have to ask is how we can evoke more reliable errors in posterior regions, and whether other mapping parameters could provide more reliable results.

Nonetheless, the aim of this study was to gather data about reproducibility of language mapping by rTMS. However, one bias of reproducibility is the spatial inconsistency of the investigated language function itself, which cannot be separated from the inaccuracy of the method. Yet, since is crucial to have data on the reproducibility of a new technique, this study has still its justification. Moreover, being the first study investigating this question, this study intended to mirror current practice of rTMS language mapping rather than examining a new rigid mapping protocol. These further aspects of reproducibility have to undergo further investigation in the future.

\section{Conclusions}

With our current protocol, interobserver and intraobserver comparison only corresponded partially. Although the technique of rTMS seems a promising method for preoperative planning as well as neuropsychological research, the current protocol needs further improvement.

\section{Abbreviations}

CCC: Concordance correlation coefficient; CPS: Cortical parcellation system; DCS: Bipolar direct cortical stimulation; fMRI: functional MRI; MEG: Magnetoencephalography; ISI: Inter-stimulus interval; nTMS: navigated transcranial magnetic stimulation; RMT: Resting Motor Threshold; rTMS: repetitive nTMS; VAS: Visual analogue scale.

\section{Competing interests}

The authors declare that they have no competing interests. The authors declare that they have no conflict of interest affecting this study. The authors 
report no conflict of interest concerning the materials or methods used in this study, or the findings specified in this paper.

\section{Authors' contributions}

NS and TH were responsible for data acquisition and drafted the manuscript. $\mathrm{AH}$ was responsible for statistical analyses, and read and approved the final manuscript. BM and FR approved and corrected the final version of the manuscript. SK handled the acquired data and performed literature research, as well as statistical analyses. SK drafted the manuscript and its final revision. SK is also responsible for concept and design. All authors read and approved the final manuscript.

\section{Authors' information}

NS and TH are medical students who are performing a considerable number of rTMS studies. All other authors are strongly involved in the treatment of brain tumors, including awake surgery, preoperative mapping, and intraoperative neuromonitoring in a specialized neurooncological center. BM is chairman, and FR is vice chairman of the department.

\section{Acknowledgements}

The study was completely financed by institutional grants from the Department of Neurosurgery.

\section{Author details}

${ }^{1}$ Department of Neurosurgery, Klinikum rechts der Isar, Technische Universität München, Ismaninger Str. 22, 81675 Munich, Germany. ${ }^{2}$ Institute of Medical Statistics and Epidemiology, Technische Universität München, Ismaninger Str. 22, 81675 Munich, Germany.

Received: 9 September 2013 Accepted: 27 November 2013 Published: 5 December 2013

\section{References}

1. Indefrey P: The spatial and temporal signatures of word production components: a critical update. Front Psychol 2011, 2:255.

2. McGraw P, Mathews VP, Wang Y, Phillips MD: Approach to functional magnetic resonance imaging of language based on models of language organization. Neuroimaging Clin N Am 2001, 11(2):343-353.

3. Hund-Georgiadis M, Lex U, von Cramon DY: Language dominance assessment by means of fMRl: contributions from task design, performance, and stimulus modality. J Magn Reson Imaging 2001, 13(5):668-675.

4. Vigneau M, Beaucousin V, Herve PY, Duffau H, Crivello F, Houde O, Mazoyer B, Tzourio-Mazoyer N: Meta-analyzing left hemisphere language areas: phonology, semantics, and sentence processing. Neuroimage 2006, 30(4):1414-1432.

5. Wheat KL, Cornelissen PL, Sack AT, Schuhmann T, Goebel R, Blomert L: Charting the functional relevance of Broca's area for visual word recognition and picture naming in Dutch using fMRI-guided TMS. Brain Lang 2013, 125(2):223-230.

6. Schuhmann T, Schiller NO, Goebel R, Sack AT: Speaking of which: dissecting the neurocognitive network of language production in picture naming. Cereb Cortex 2012, 22(3):701-709.

7. Ojemann G, Ojemann J, Lettich E, Berger M: Cortical language localization in left, dominant hemisphere. An electrical stimulation mapping investigation in 117 patients. J Neurosurg 1989, 71(3):316-326.

8. Haglund MM, Berger MS, Shamseldin M, Lettich E, Ojemann GA: Cortical localization of temporal lobe language sites in patients with gliomas. Neurosurgery 1994, 34(4):567-576. discussion 576.

9. Sanai N, Mirzadeh Z, Berger MS: Functional outcome after language mapping for glioma resection. N Engl J Med 2008, 358(1):18-27.

10. Chang EF, Wang DD, Perry DW, Barbaro NM, Berger MS: Homotopic organization of essential language sites in right and bilateral cerebral hemispheric dominance. J Neurosurg 2011, 114(4):893-902.

11. Pascual-Leone A, Gates JR, Dhuna A: Induction of speech arrest and counting errors with rapid-rate transcranial magnetic stimulation. Neurology 1991, 41(5):697-702.

12. Wassermann EM, Blaxton TA, Hoffman EA, Berry CD, Oletsky H, Pascual-Leone A, Theodore WH: Repetitive transcranial magnetic stimulation of the dominant hemisphere can disrupt visual naming in temporal lobe epilepsy patients. Neuropsychologia 1999, 37(5):537-544.
13. Epstein CM, Lah JJ, Meador K, Weissman JD, Gaitan LE, Dihenia B: Optimum stimulus parameters for lateralized suppression of speech with magnetic brain stimulation. Neurology 1996, 47(6):1590-1593.

14. Lioumis P, Zhdanov A, Makela N, Lehtinen H, Wilenius J, Neuvonen T, Hannula H, Deletis V, Picht T, Makela JP: A novel approach for documenting naming errors induced by navigated transcranial magnetic stimulation. J Neurosci Methods 2012, 204(2):349-354.

15. Picht T, Krieg SM, Sollmann N, Rosler J, Niraula B, Neuvonen T, Savolainen $P$, Lioumis P, Makela JP, Deletis V, et al: A comparison of language mapping by preoperative navigated transcranial magnetic stimulation and direct cortical stimulation during awake surgery. Neurosurgery 2013, 72(5):808-819.

16. Sollmann N, Picht T, Makela JP, Meyer B, Ringel F, Krieg SM: Navigated transcranial magnetic stimulation for preoperative language mapping in a patient with a left frontoopercular glioblastoma. J Neurosurg 2013, 118(1):175-179.

17. Sollmann N, Hauck T, Obermuller T, Hapfelmeier A, Meyer B, Ringel F, Krieg SM: Inter- and intraobserver variability in motor mapping of the hotspot for the abductor policis brevis muscle. BMC Neurosci 2013, 14(1):94.

18. Forster MT, Limbart M, Seifert V, Senft C: Test-Retest-Reliability of Navigated Transcranial Magnetic Stimulation of the Motor Cortex. Neurosurgery 2013, [Epub ahead of print].

19. Krieg SM, Sollmann N, Hauck T, Ille S, Foerschler A, Meyer B, Ringel F: Functional language shift to the right hemisphere in patients with language-eloquent brain tumors. PLoS One 2013, 8(9):e75403.

20. Salmelin R, Helenius P, Service E: Neurophysiology of fluent and impaired reading: a magnetoencephalographic approach. J Clin Neurophysio/ 2000, 17(2):163-174.

21. Krieg SM, Shiban E, Buchmann N, Gempt J, Foerschler A, Meyer B, Ringel F: Utility of presurgical navigated transcranial magnetic brain stimulation for the resection of tumors in eloquent motor areas. J Neurosurg 2012, 116(5):994-1001.

22. Picht T, Mularski S, Kuehn B, Vajkoczy P, Kombos T, Suess O: Navigated transcranial magnetic stimulation for preoperative functional diagnostics in brain tumor surgery. Neurosurgery 2009, 65(6 Suppl):93-98.

23. Epstein CM: Transcranial magnetic stimulation: language function. J Clin Neurophysiol 1998, 15(4):325-332.

24. Corina DP, Loudermilk BC, Detwiler L, Martin RF, Brinkley JF, Ojemann G: Analysis of naming errors during cortical stimulation mapping: implications for models of language representation. Brain Lang 2010, 115(2):101-112.

25. Corina DP, Gibson EK, Martin R, Poliakov A, Brinkley J, Ojemann GA: Dissociation of action and object naming: evidence from cortical stimulation mapping. Hum Brain Mapp 2005, 24(1):1-10.

26. Lin LI: A concordance correlation coefficient to evaluate reproducibility. Biometrics 1989, 45(1):255-268.

27. Talacchi A, Santini B, Casartelli M, Monti A, Capasso R, Miceli G: Awake surgery between art and science. Part II: language and cognitive mapping. Funct Neurol 2013, 28(3):223-229.

28. Duffau H, Moritz-Gasser S, Mandonnet E: A re-examination of neural basis of language processing: proposal of a dynamic hodotopical model from data provided by brain stimulation mapping during picture naming. Brain Lang 2013, [Epub ahead of print].

29. Ungerleider LG, Doyon J, Karni A: Imaging brain plasticity during motor skill learning. Neurobiol Learn Mem 2002, 78(3):553-564.

30. Pascual-Leone A, Nguyet D, Cohen LG, Brasil-Neto JP, Cammarota A, Hallett M: Modulation of muscle responses evoked by transcranial magnetic stimulation during the acquisition of new fine motor skills. J Neurophysiol 1995, 74(3):1037-1045.

31. Rijntjes M, Weiller C: Recovery of motor and language abilities after stroke: the contribution of functional imaging. Prog Neurobio/ 2002, 66(2):109-122

32. Munte TF, Altenmuller $E$, Jancke L: The musician's brain as a model of neuroplasticity. Nat Rev Neurosci 2002, 3(6):473-478.

33. Draganski B, Gaser C, Kempermann G, Kuhn HG, Winkler J, Buchel C, May A: Temporal and spatial dynamics of brain structure changes during extensive learning. J Neurosci 2006, 26(23):6314-6317.

doi:10.1186/1471-2202-14-150

Cite this article as: Sollmann et al:: Intra- and interobserver variability of language mapping by navigated transcranial magnetic brain stimulation. BMC Neuroscience 2013 14:150. 